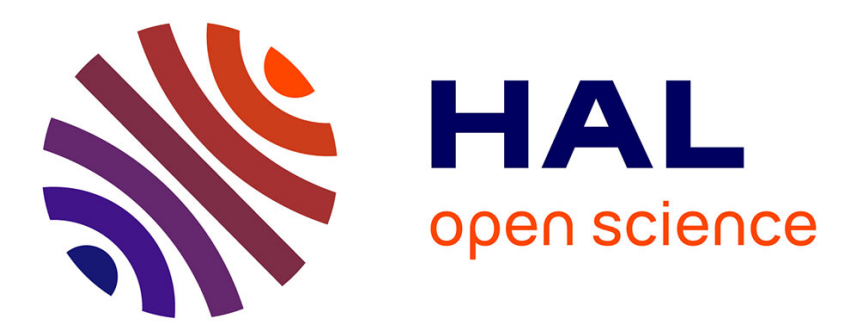

\title{
Light scattering by periodic rough surfaces: equivalent jump conditions
}

Bruno Gallas, Agnes Maurel, Jean-Jacques Marigo, Abdelwaheb Ourir

\section{To cite this version:}

Bruno Gallas, Agnes Maurel, Jean-Jacques Marigo, Abdelwaheb Ourir. Light scattering by periodic rough surfaces: equivalent jump conditions. Journal of the Optical Society of America. A Optics, Image Science, and Vision, 2017, 34 (12), pp.2181-2188 10.1364/JOSAA.34.002181 . hal-01657085

\section{HAL Id: hal-01657085 \\ https://hal-polytechnique.archives-ouvertes.fr/hal-01657085}

Submitted on 6 Dec 2017

HAL is a multi-disciplinary open access archive for the deposit and dissemination of scientific research documents, whether they are published or not. The documents may come from teaching and research institutions in France or abroad, or from public or private research centers.
L'archive ouverte pluridisciplinaire HAL, est destinée au dépôt et à la diffusion de documents scientifiques de niveau recherche, publiés ou non, émanant des établissements d'enseignement et de recherche français ou étrangers, des laboratoires publics ou privés. 


\title{
Light scattering by periodic rough surfaces - equivalent jump conditions
}

\author{
Bruno Gallas ${ }^{1}$, Agnès Maurel ${ }^{2}$, Jean -Jacques Marigo ${ }^{3}$, And Abdelwaheb Ourir ${ }^{2}$ \\ ${ }^{1}$ Institut des NanoSciences de Paris, 4 place Jussieu, 75005 Paris, France \\ ${ }^{2}$ Institut Langevin, 1 rue Jussieu, 75005 Paris, France \\ ${ }^{3}$ LMS, Ecole Polytechnique, Route de Saclay, 91128 Palaiseau, France \\ *Corresponding author: agnes.maurel@espci.fr
}

Compiled August 16, 2017

\begin{abstract}
We present an interface model based on two-scale homogenization to predict the coherent scattering of light by a periodic rough interface between air and a dielectric. Contrary to previous approaches where the roughnesses are replaced by a layer filled with an equivalent medium, our modeling yields effective jump conditions applying across the region containing the roughnesses. The validity of the model is inspected by comparison with direct numerics and with experimental measurements on a air/silicium rough interface near the Brewster angle. It is shown that the interface model reproduces accurately the shift in the Brewster phenomenon without any ajustable parameter, which is of practical importance in retrieval methods to get thickness or filling fraction with reliable physical values.
\end{abstract}

(C) 2017 Optical Society of America

OCIS codes: $\quad 240.5770 ; 050.2065 ; 050.6624 ; 310.5448$.

http://dx.doi.org/10.1364/ao.XX.XXXXXX

\section{INTRODUCTION}

In 1990, Saillard and Maystre reported a quantitative numerical study of the coherent scattering of light by a rough air-dielectric interface [1]. Their observation of an angular shift of the Brewster angle was explained on the basis of physical arguments in [2,3] and studied for random roughnesses using stochastic approaches based on the resolution of the Dyson equation satisfied by the ensemble averaged Green's function [4, 5], for a review see [6]. It has been confirmed that the reflection coefficient of light impinging a rough interface no longer vanishes but has a minimum that is slightly shifted from the position of the Brewster angle. The angle realizing the minimum in reflection is often termed "pseudo- Brewster angle", and it depends on the rms height surface and on the correlation length of the roughnesses. Because this perturbation approach is developed within the Rayleigh hypothesis, slightly rough surfaces are considered with small rms height compared to the incident wavelength (a classical bound for the correlation length and for the rms heigh is one third of the wavelength).

Alongside the development of these stochastic approaches, works have been devoted to the case of periodic or quasi periodic roughnesses. In the low frequency regime, a natural tool to describe the interaction of light with a subwavelength structure is the homogenization. As developed in [7,8], the homogenization provides an equivalent layer of the same thickness than the roughnesses filled with an effective medium, in general inhomogeneous along the roughness direction. While random roughnesses have been studied in the limit of small roughness height, this homogenization approach is restricted to the opposite limit of deep roughnesses. This is because such homogenization (in the bulk) requires that the waves propagate over typically one, or more, wavelength in the structured medium. Thus, if the periodicity of the structure has to be small compared to wavelength, its height has to be of the same order or larger than the wavelength, and this is a limitation of these approaches, including the well known Bruggeman and MaxwellGarnett mixture formula [9]. In the following, we shall term these models "layer models".

In this work, we present an "interface model" based on a homogenization procedure adapted for slightly rough surfaces. While the layer models provide effective parameters reflecting the propagation properties in the roughnesses, the interface model provides parameters which encapsulate the effect of the evanescent field excited at the edges of the roughnesses. Note that these boundary layer effects exist also for large roughnesses but they can be in general neglected. More specifically, with $h$ the period and $e$ the height of the 


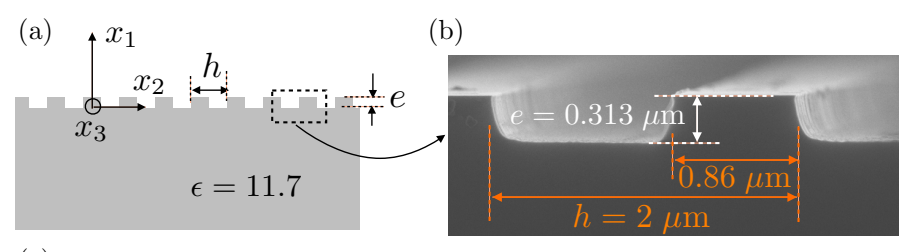

(c)

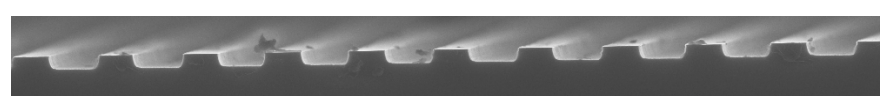

Fig. 1. (a) Periodic rough interface between air and a dielectric, (b) SEM view of the realized sample with Silicium, (c) shows a lager view of the interface.

roughnesses, boundary layer effects are of the order $(k h)$, with $k$ the wavenumber while the propagation effects are of the order $(k e)$. Subwavelength structurations, as roughnesses, satisfy $k h \ll 1$; next, for deep roughnesses $k e=O(1) \gg k h$, thus the boundary layer effects are negligible compared to the propagation effects; however, when ( $k e)$ decreases, they may become of the same order and eventually dominant. A discussion on the validity of the homogenization in the bulk (layer model) compared to the homogenization of the interface (interface model) can be found in $[10,11]$ in the case of arrays made of a perfect conductor.

The interface model is presented in Section 2. For transverse magnetic polarization of light in two dimensions, we derive the effective jump conditions across an equivalent interface of thickness $e$, Eqs. (22) which involve two parameters $(\mathcal{B}, \mathcal{C})$ characteristic of the roughnesses (it is worth noting that the interior of the interface is not questioned). The reflection coefficient for an incident plane wave at oblique incidence is deduced, Eq. (26). For comparison, we give the reflection coefficient in a layer model, (31), similar to that given by the anisotropic Bruggeman model. In Section 4, the validity of the models is inspected by comparison with direct numerical calculations based on modal method [12] and with experimental measurements. The results show that the interface model is able to accurately describe the scattering properties of small roughnesses when their actual geometry is considered in the model. In comparison layer models overestimate the effect of the roughnesses, leading to a larger shift in the Brewster phenomenom. The discrepancy between layer and interface models is discussed in Section 5 in the light of calculations performed for varying roughness thicknesses.

\section{HOMOGENIZATION OF PERIODIC SLIGHTLY ROUGH SURFACE}

We consider a periodic rough interface $x_{1}=f\left(x_{2}\right)$ invariant along $x_{3}$ separating the air from a dielectric region with relative permittivity $\varepsilon_{\mathrm{r}}$. We shall see that within the framework of homogenization theory, the periodic roughnesses of thickness $e$ and spacing $h$ can be replaced by an equivalent interface of same thickness across which effective jump conditions apply. We start with the wave equation written for the magnetic field $H(\mathbf{x})\left(\mathbf{x}=\left(x_{1}, x_{2}\right)\right)$ being polarized along $\mathbf{e}_{3}$, which reads as

$$
\operatorname{div}\left[\frac{1}{\varepsilon(\mathbf{x})} \nabla H(\mathbf{x})\right]+k^{2} H(\mathbf{x})=0,
$$

where $\varepsilon(\mathbf{x})$ is the relative permittivity which depends on space; we denote $\varepsilon(\mathbf{x})=\varepsilon^{-}=\varepsilon_{\mathrm{r}}$ in the dielectric and $\varepsilon(\mathbf{x})=\varepsilon^{+}=1$ in the air, and $k=\sqrt{\varepsilon_{0} \mu_{0}} \omega$ is the wavenumber in the air. To Eq. (1), we have to associate boundary conditions. The boundary conditions at the air/dielectric interface are the continuities of $H$ and of $1 / \varepsilon \nabla H \cdot \mathbf{n}$. Finally, once the incident wave has been defined, radiation conditions apply for the scattered field; for the time being, we do not need to specify them.

For simplicity, we shall work with the field $C$ such that (1) is written

$$
\operatorname{div} \boldsymbol{C}(\mathbf{x})+k^{2} H(\mathbf{x})=0, \quad \boldsymbol{C}(\mathbf{x}) \equiv \frac{1}{\varepsilon(\mathbf{x})} \nabla H(\mathbf{x}),
$$

with boundary conditions being now the continuities of $H$ and of $C . n$ at the air/dielectric interface. It is easy to see that the electric field $\mathbf{E}=\left(E_{1}, E_{2}, 0\right)$ can be deduced from $C$, with

$$
C_{1}=-\mathrm{i} \omega E_{2}, \quad C_{2}=\mathrm{i} \omega E_{1}
$$

(the continuity of the normal component of $C$ corresponds to the continuity of the tangential component of $\mathbf{E}$ ).

As in the classical homogenization, we shall work using two systems of coordinates. Without loss of generality, we consider

$$
k=O(1), \quad \eta=h \ll 1,
$$

and we have $e=O(h)$. Thus $\mathbf{x}=\left(x_{1}, x_{2}\right)$ is the coordinate at the macroscale $1 / k$, and we define the coordinate

$$
\mathbf{y}=\mathbf{x} / h,
$$

the coordinate at the microscale $h$. Fig. 2 shows the resulting rescaling in $\mathbf{y}$ coordinates of the elementary cell $Y=\left(-y_{1}^{m}, y_{1}^{m}\right) \times$ $(-1 / 2,1 / 2)$; the value of $y_{1}^{m}>e /(2 h)$ is incidental, since we shall consider the limit $y_{1}^{m} \rightarrow \infty$ in practice. We define also $\mathrm{Y}^{+}$and $\mathrm{Y}^{-}$ the subregions of $Y$ occupied by the air and by the dielectric respectively. 


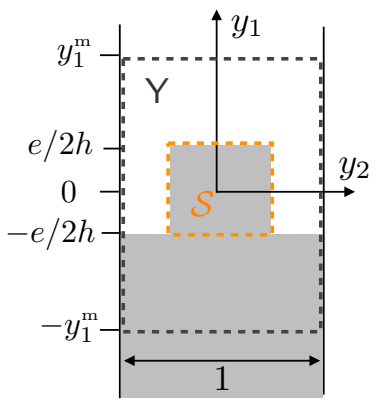

Fig. 2. Elementary cell in $\left(y_{1}, y_{2}\right)$ coordinates. $Y=\left(-y_{1}^{m}, y_{1}^{m}\right) \times(-1 / 2,1 / 2)$ (and the limit $y_{1}^{m} \rightarrow \infty$ will be considered in practice); $\mathrm{Y}^{+}$and $\mathrm{Y}^{-}$are the subregions of $\mathrm{Y}$ occupied by the air $\left(\varepsilon^{+}=1\right)$ and by the dielectric $\left(\varepsilon^{-}=\varepsilon_{\mathrm{r}}\right)$ respectively.

\section{A. Expansions of the solution in the near and far fields and matching conditions}

\section{A.1. Near and far field expansions}

The idea is to define two different expansions of $(H, C)$ in the far field and in the near field of the roughnesses. In the far field, we use

$$
H=H^{0}(\mathbf{x})+\eta H^{1}(\mathbf{x})+\cdots, \quad C=C^{0}(\mathbf{x})+\eta \boldsymbol{C}^{1}(\mathbf{x})+\cdots,
$$

with the terms in the expansions depending on $\mathbf{x}$ only which is adapted to describe the variations of the propagating waves (they vary on the wavelength scale $1 / k$ ). In the near field, we use

$$
H=h^{0}\left(\mathbf{y}, x_{2}\right)+\eta h^{1}\left(\mathbf{y}, x_{2}\right)+\cdots, \quad C=\mathbf{c}^{0}\left(\mathbf{y}, x_{2}\right)+\eta \mathbf{c}^{1}\left(\mathbf{y}, x_{2}\right) \cdots
$$

with a dependence on $\mathbf{y}$ adapted to describe the variations of the non propagating waves (or evanescent waves) at the scale $h$ and a dependence on $x_{2}$ adapted to describe the variations of the propagating waves along the roughnesses. The terms $h^{n}$ and $c^{n}, n=0, \cdots$, are assumed to be periodic with respect $y_{2}$. This tells us that the field at the microscale is repeated from one cell to the other while long scale variations, at the scale of the wavelength, are handled by $x_{2}$. Eventually, the Eqs. (2) will be written at the orders $\eta^{-1}$ and $\eta^{0}$ in the near field, and at the orders $\eta^{0}$ and $\eta^{1}$ in the far field, using the differential operators which take the forms

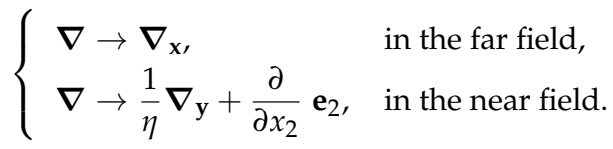

\section{A.2. Matching conditions}

From a unique problem, we have build two applying in two different regions. Thus, we have to associate to each problem boundary conditions in order that they are well-posed. The boundary conditions on the roughnesses apply in the near field only (at each order in $\eta$ ) while the radiation conditions apply in the far field only. Thus, there are missing boundary conditions in the near field when $y_{1} \rightarrow \pm \infty$ which are the equivalent of the radiation conditions; reversely there are missing conditions in the far field when $x_{1} \rightarrow 0^{ \pm}$, which correspond to the equivalent conditions that we are looking for (these conditions encapsulate the effect of the roughnesses). These missing boundary conditions are provided simultaneously by the so-called matching conditions which ensure the continuity of $H$ and $C$ in an intermediate region where the evanescent field can be considered as negligible, typically when $\left|x_{1}\right| \sim O(\sqrt{\eta}) \rightarrow 0$ and thus $\left|y_{1}\right| \sim O(1 / \sqrt{\eta}) \rightarrow+\infty$. We thus write that $H^{0}(\mathbf{x})+\eta H^{1}(\mathbf{x}) \sim h^{0}\left(\mathbf{y}, x_{2}\right)+\eta h^{1}\left(\mathbf{y}, x_{2}\right)$ in this intermediate region, and identify the limits at each order in $\eta$. Owing to the Taylor expansions $H^{0}\left(x_{1}, x_{2}\right)=H^{0}\left(0^{+}, x_{2}\right)+x_{1} \partial_{x_{1}} H^{0}\left(0^{+}, x_{2}\right)+\cdots=$ $H^{0}\left(0^{+}, x_{2}\right)+\eta y_{1} \partial_{x_{1}} H^{0}\left(0^{+}, x_{2}\right)+\cdots$ (same for $\left.C^{0}\right)$, we get at the order 0

$$
H^{0}\left(0^{ \pm}, x_{2}\right)=\lim _{y_{1}^{m} \rightarrow+\infty} h^{0}\left( \pm y_{1}^{m}, y_{2}, x_{2}\right), \quad C^{0}\left(0^{ \pm}, x_{2}\right)=\lim _{y_{1}^{m} \rightarrow+\infty} \mathbf{c}^{0}\left( \pm y_{1}^{m}, y_{2}, x_{2}\right),
$$

and at the order 1 ,

$$
\left\{\begin{array}{l}
H^{1}\left(0^{ \pm}, x_{2}\right)=\lim _{y_{1}^{m} \rightarrow+\infty}\left[h^{1}\left( \pm y_{1}^{m}, y_{2},, x_{2}\right) \mp y_{1}^{m} \frac{\partial H^{0}}{\partial x_{1}}\left(0^{ \pm}, x_{2}\right)\right], \\
C^{1}\left(0^{ \pm}, x_{2}\right)=\lim _{y_{1}^{m} \rightarrow+\infty}\left[\mathbf{c}^{1}\left( \pm y_{1}^{m}, y_{2}, x_{2}\right) \mp y_{1}^{m} \frac{\partial C^{0}}{\partial x_{1}}\left(0^{ \pm}, x_{2}\right)\right] .
\end{array}\right.
$$

Finally, something has to be said on the variations of $\varepsilon$. In the far field regions $x_{1}<0$ and $x_{1}>0, \varepsilon=\varepsilon^{-}$and $\varepsilon=\varepsilon^{+}$are constant since the far fields do not contain the roughness region. The variations of $\varepsilon$ concern the near field only, and being periodic, we simply have $\varepsilon(\mathbf{y})$ with no long scale variation of $\varepsilon$ with $x_{2}$ and with

$$
\lim _{y_{1}^{m} \rightarrow+\infty} \varepsilon\left( \pm y_{1}^{m}, y_{2}\right)=\varepsilon^{ \pm}
$$




\section{B. The leading and first order solutions and the associated jump conditions}

\section{B.1. The solution at the order 0}

In the near field, (2) gives $\nabla_{\mathbf{y}} h^{0}=\mathbf{0}$ at leading order, in $\eta^{-1}$. It follows that $h^{0}$ does not depend on $\mathbf{y}$ and, from the first matching condition in (9), we deduce that

$$
h^{0}\left(x_{2}\right)=H^{0}\left(0^{ \pm}, x_{2}\right) .
$$

From (2), we also have $\operatorname{div}_{\mathbf{y}} \mathbf{c}^{0}=0$. Integrating this equation over $\mathrm{Y}$ and owing to the continuities $\mathbf{c}^{0} \cdot \mathbf{n}$ at the air/dielectric interface and to the periodicity of $\mathrm{c}^{0}$ w.r.t $y_{2}$, we get $\int \mathrm{d} y_{2} c_{1}^{0}\left(y_{1}^{m}, y_{2}, x_{2}\right)=\int \mathrm{d} y_{2} c_{1}^{0}\left(-y_{1}^{m}, y_{2}, x_{2}\right)$. Then, it is sufficient to take the limit $y_{1}^{m} \rightarrow+\infty$ in the second matching condition of (9) to get

$$
C_{1}^{0}\left(0^{+}, x_{2}\right)=C_{1}^{0}\left(0^{-}, x_{2}\right) \text {. }
$$

At the dominant order, the roughnesses are not visible: the rough interface is equivalent to a flat interface between the air and the dielectric, with the continuities of $H^{0}$ and of $C_{1}^{0}$, which correspond to the usual continuities of the magnetic field and of the tangential component of the electric field from (3). To capture the effect of the roughnesses, we need to go to the next order.

\section{B.2. Elementary problems}

Before going to the next order, elementary problems have to be defined, which will make the effective interface parameters to appear. To that aim, we focus on the problem satisfied by $h^{1}$, which, from (2), reads as

$$
\left\{\begin{array}{l}
\operatorname{div}_{\mathbf{y}} \mathbf{c}^{0}=0, \text { with } \mathbf{c}^{0}=\frac{1}{\varepsilon(\mathbf{y})}\left[\boldsymbol{\nabla}_{\mathbf{y}} h^{1}+\frac{\partial H^{0}}{\partial x_{2}}\left(0, x_{2}\right) \mathbf{e}_{2}\right], \\
\lim _{y_{1} \rightarrow \pm \infty} \nabla_{\mathbf{y}} h^{1}=\varepsilon^{ \pm} C_{1}^{0}\left(0, x_{2}\right) \mathbf{e}_{1},
\end{array}\right.
$$

with boundary conditions being the continuities of $h^{1}$ and of $c^{0} \cdot \mathbf{n}$ at each air/dielectric interface. To get (14), we have used $C=$ $1 / \varepsilon \nabla_{\mathbf{x}} H$ written at the order $\eta^{0}$ for the near field solution, and $h^{0}\left(x_{2}\right)=H^{0}\left(0, x_{2}\right)$ from (12). We also used the matching conditions $\mathbf{c}^{0}\left( \pm \infty, y_{2}, x_{2}\right)=C_{1}^{0}\left(0, x_{2}\right) \mathbf{e}_{1}+1 / \varepsilon^{ \pm} \partial_{x_{2}} H^{0}\left(0, x_{2}\right) \mathbf{e}_{2}$ in (14) to get the limits of $\nabla_{\mathbf{y}} h^{1}$. The problem (14) is linear $w . r . t \partial_{x_{2}} H^{0}\left(0, x_{2}\right)$ and $C_{1}^{0}\left(0, x_{2}\right)$, thus we can write

$$
h^{1}\left(\mathbf{y}, x_{2}\right)=C_{1}^{0}\left(0, x_{2}\right) h^{(1)}(\mathbf{y})+\frac{\partial H^{0}}{\partial x_{2}}\left(0, x_{2}\right) h^{(2)}(\mathbf{y})+\hat{h}\left(x_{2}\right),
$$

and $h^{1}$ is solution of the problem (i) for any incident wave (thus any values of $\partial_{x_{2}} H^{0}\left(0, x_{2}\right)$ and $\left.C_{1}^{0}\left(0, x_{2}\right)\right)$ if $h^{(1)}$ satisfies

$$
\operatorname{div}\left[\frac{1}{\varepsilon(\mathbf{y})} \nabla h^{(1)}\right]=0, \quad \lim _{y_{1} \rightarrow \pm \infty} \nabla h^{(1)}=\varepsilon^{ \pm} \mathbf{e}_{1},
$$

with $h^{(1)}$ being a $y_{2}$-periodic and continuous and with $1 / \varepsilon(\mathbf{y}) \nabla h^{(1)} \cdot \mathbf{n}$ being continuous at the air/dielectric interface, and (ii) if $h^{(2)}$ satisfies

$$
\operatorname{div}\left[\frac{1}{\varepsilon(\mathbf{y})} \nabla\left(h^{(2)}+y_{2}\right)\right]=0, \quad \lim _{y_{1} \rightarrow \pm \infty} \nabla h^{(2)}=\mathbf{0},
$$

with $h^{(2)}$ being a $y_{2}$-periodic and continuous and with $1 / \varepsilon(\mathbf{y}) \boldsymbol{\nabla}\left(h^{(2)}+y_{2}\right) \cdot \mathbf{n}$ being continuous at the air/dielectric interface. The so-built elementary solutions $\left(h^{(1)}, h^{(2)}\right)$ are defined up to a constant and can be written

$$
h^{(1)}(\mathbf{y})=\left\{\begin{array}{ll}
\varepsilon^{-} y_{1}+\mathcal{B}^{-}+h_{\mathrm{ev}}^{(1)}(\mathbf{y}), & y_{1}<0, \\
\varepsilon^{+} y_{1}+\mathcal{B}^{+}+h_{\mathrm{ev}}^{(1)}(\mathbf{y}), & y_{1}>0 .
\end{array} \quad \text { and } \quad h^{(2)}(\mathbf{y})=h_{\mathrm{ev}}^{(2)}(\mathbf{y})\right.
$$

where $h_{\mathrm{ev}}^{(1)}$ and $\left.h_{\mathrm{ev}}^{(2)}\right)$ are evanescent fields vanishing at $y_{1} \rightarrow \pm \infty$. Indeed, for symmetric shapes of roughnesses, $h^{(2)}$ is an odd function of $y_{2}$, while $h^{(1)}$ is even, thus this latter has possibly two different constants at $y_{1} \rightarrow \pm \infty$. The evanescent fields, as the constant $\mathcal{B}^{+}-\mathcal{B}^{-}$, depend on the shape of the roughnesses and on $\varepsilon_{\mathrm{r}}$ and the problems have to be solved numerically; we shall see in the following section that these elementary solutions make the interface parameters to appear.

\section{B.3. Solution at order 1}

The limits $H^{1}\left(0^{ \pm}, x_{2}\right)$ directly follows from the first matching condition in (10), using (15) along with (18). Specifically, using that $\partial_{x_{1}} H^{0}\left(0^{ \pm}, x_{2}\right)=\varepsilon^{ \pm} C_{1}^{0}\left(0, x_{2}\right)$, we easily obtain that

$$
\left\{\begin{array}{l}
H^{1}\left(0^{+}, x_{2}\right)=\mathcal{B}^{+} C_{1}^{0}\left(0, x_{2}\right)+\hat{h}\left(x_{2}\right) \\
H^{1}\left(0^{-}, x_{2}\right)=\mathcal{B}^{-} C_{1}^{0}\left(0, x_{2}\right)+\hat{h}\left(x_{2}\right)
\end{array}\right.
$$

Now, we need to determine the jump in $C_{1}^{1}$. To do so, we start by integrating over $Y$ the first equation of Eqs. (2) written at order 0 in $\eta$, that is

$$
\int_{Y} d \mathbf{y}\left[\operatorname{div}_{\mathbf{y}} \mathbf{c}^{1}+\frac{\partial c_{2}^{0}}{\partial x_{2}}+H^{0}\left(0, x_{2}\right)\right]=0 .
$$


We have $\int_{\mathbf{Y}} \operatorname{div}_{\mathbf{y}} \mathbf{c}^{1}=c_{1}^{1}\left(y_{1}^{m}\right)-c_{1}^{1}\left(-y_{1}^{m}\right)$ using the continuity of $\mathbf{c}^{1} \cdot \mathbf{n}$ and the periodicity of $\mathbf{c}^{1}$. Next, with $c_{2}^{0}=1 / \varepsilon\left[\partial_{y_{2}} h^{1}+\partial_{x_{2}} H^{0}\left(0, x_{2}\right)\right]$ from (14), and using (15), it is straightforward to obtain that

$$
C_{1}^{1}\left(0^{+}, x_{2}\right)-C_{1}^{1}\left(0^{-}, x_{2}\right)=-\left[\frac{e}{h}(1-f)\left(\frac{1}{\varepsilon^{+}}-\frac{1}{\varepsilon^{-}}\right)+\int_{Y} \frac{\mathrm{d} \mathbf{y}}{\varepsilon(\mathbf{y})} \frac{\partial h^{(2)}}{\partial y_{2}}(\mathbf{y})\right] \frac{\partial^{2} H^{0}}{\partial x_{2}^{2}}\left(0, x_{2}\right),
$$

with $f$ the filling fraction of the roughnesses, thus the surface of the roughness equal to $\mathcal{S}=e h f$. In the matching conditions (10), we used that $\left(1 / \varepsilon^{ \pm}\right) \partial_{x_{2}^{2}}^{2} H^{0}+k^{2} H^{0}=-\partial_{x_{1}} C_{1}^{0}$ at $x_{1}=0^{ \pm}$respectively. Eventually, we also used that $h^{(1)}$ is even, from which the integral of $\partial_{y_{2}} h^{(1)}$ over $Y$ vanishes.

\section{B.4. Final jump conditions and interface parameters}

To get the final form of the jump conditions, we need two additional steps. First, the conditions (12)-(13) at the order 0 and the conditions (19)-(21) at the order 1 are used to write the jump conditions on $\left(H^{0}+\eta H^{1}\right)$ and on $\left(C_{1}^{0}+\eta C_{1}^{1}\right)$ which admit the same expansions as $H$ and $C$ up to $O\left(\eta^{2}\right)$, and at this stage, the jumps have been expressed across a zero thickness interface, namely between $x_{1}=0^{-}$and $x_{1}=0^{+}$. It has been previously discussed that restoring the thickness of the actual interface improves the accuracy of the model, see $e . g$. $[13,14]$. This is done by "enlarging" the equivalent interface; to do that, we define $H^{ \pm}=H\left( \pm e / 2, x_{2}\right)$, and $\bar{H}=1 / 2\left(H^{+}+H^{-}\right)+O(e)$ with $e=O(\eta)$ (the same for $\left.C^{ \pm}\right)$. Next, the jump $\llbracket H \rrbracket \equiv H^{+}-H^{-}=H\left(0^{+}, x_{2}\right)-H\left(0^{-}, x_{2}\right)+e \frac{2}{\partial_{x_{1}} H}+O\left(e^{2}\right)$, where we have used Taylor expansions of $H\left( \pm e / 2, x_{2}\right)$. The jump conditions at the new equivalent interface of thickness $e$ finally read as

$$
\left\{\begin{array}{l}
H^{+}-H^{-}=\frac{h \mathcal{B}}{2}\left[\frac{1}{\varepsilon^{-}} \frac{\partial H^{-}}{\partial x_{1}}+\frac{1}{\varepsilon^{+}} \frac{\partial H^{+}}{\partial x_{1}}\right], \\
\frac{1}{\varepsilon^{+}} \frac{\partial H^{+}}{\partial x_{1}}-\frac{1}{\varepsilon^{-}} \frac{\partial H^{-}}{\partial x_{1}}=-\frac{h \mathcal{C}}{2}\left[\frac{\partial^{2} H^{-}}{\partial x_{2}^{2}}+\frac{\partial^{2} H^{+}}{\partial x_{2}^{2}}\right]-\frac{e k^{2}}{2}\left[H^{-}+H^{+}\right],
\end{array}\right.
$$

with $(\mathcal{B}, \mathcal{C})$ the interface parameters defined by

$$
\left\{\begin{aligned}
\mathcal{B} & =\mathcal{B}^{+}-\mathcal{B}^{-}+\frac{e}{2 h}\left(\varepsilon+\varepsilon^{+}\right) \\
\mathcal{C} & =\int_{Y} \frac{\mathrm{d} \mathbf{y}}{\varepsilon(\mathbf{y})} \frac{\partial h^{(2)}}{\partial y_{2}}+\frac{e}{h}\left(\frac{f}{\varepsilon^{+}}+\frac{1-f}{\varepsilon^{-}}\right) .
\end{aligned}\right.
$$

\section{REFLECTION BY A PERIODIC ROUGH INTERFACE}

In this section, we give the reflection coefficient of an incident plane wave predicted by our interface model. For comparison, we also give the reflection coefficient predicted by a classical layer model, corresponding to the two-scale homogenization in the bulk of the roughnesses.

\section{A. The interface model for roughnesses}

Using the Fresnel notations, and with a time dependence $e^{\mathrm{i} \omega t}$, the solution in the homogenized problem reads as

$$
H\left(x_{1}, x_{2}\right)= \begin{cases}{\left[e^{\mathrm{i} \alpha\left(x_{1}+e / 2\right)}-r e^{-\mathrm{i} \alpha\left(x_{1}+e / 2\right)}\right] e^{-\mathrm{i} \beta x_{2}},} & x_{1}>e / 2, \\ t e^{\mathrm{i} \gamma\left(x_{1}-e / 2\right)} e^{-\mathrm{i} \beta x_{2},} & x_{1}<-e / 2,\end{cases}
$$

with

$$
\alpha=k \cos \theta, \quad \beta=k \sin \theta, \quad \text { and } \gamma=k \sqrt{\varepsilon-\sin ^{2} \theta} .
$$

It is worth noting that the region $-e / 2<x_{1}<e / 2$ is not considered since the jump conditions (22) do not question this region and allows to determine the scattering coefficients $\left(r_{\mathrm{TM}}, t_{\mathrm{TM}}\right)$ unambiguously (the homogenized problem is simply one dimensional along $x_{1}$ ). The reflection coefficient reads as

$$
\begin{gathered}
r_{\mathrm{TM}}=\frac{z_{1}-z_{2}}{z_{1}+z_{2}}, \\
\text { with }\left\{\begin{array}{l}
z_{1}=\frac{\gamma}{\varepsilon}-\left(h \mathcal{C} \beta^{2}-e k^{2}\right)\left(\mathrm{i}-\frac{h \mathcal{B}}{4} \frac{\gamma}{\varepsilon}\right), \\
z_{2}=\alpha\left[1+\mathrm{i} h \mathcal{B} \frac{\gamma}{\varepsilon}+\left(h \mathcal{C} \beta^{2}-e k^{2}\right) \frac{h \mathcal{B}}{4}\right] .
\end{array}\right.
\end{gathered}
$$

At the dominant order, we recover the reflection coefficient of a flat interface $r_{0}=\left(\frac{\gamma}{\varepsilon}-\alpha\right) /\left(\frac{\gamma}{\varepsilon}+\alpha\right)$ vanishing at the Brewster angle

$$
\theta_{\mathrm{B}}=\operatorname{atan}(\sqrt{\varepsilon}) \text {. }
$$

Then, it is easy to see that the reflected field does not vanish near the Brewster angle but presents a minimum $r_{\min }$ at $\theta=\theta_{\mathrm{B}}+O\left(\eta^{2}\right)$, with

$$
r_{\min }=\mathrm{i} \frac{k h}{2 \sqrt{\varepsilon+1}}\left[(1+\varepsilon) \frac{e}{h}-\mathcal{B}-\varepsilon \mathcal{C}\right] .
$$




\section{B. The classical layer model}

When the classical homogenization is sought, the case of layered media yields a simple solution in the form of an equivalent homogeneous and anisotropic medium [15] and e.g. [16, 17] for practical applications. The anisotropic medium is characterized by $\left(\varepsilon_{\|,}, \varepsilon_{\perp}\right)$ given by

$$
\varepsilon_{\|}=\frac{f}{\varepsilon^{+}}+\frac{(1-f)}{\varepsilon^{-}}, \quad \varepsilon_{\perp}=f \varepsilon^{+}+(1-f) \varepsilon^{-} .
$$

When considering the problem of the scattering of an incident plane wave for a layer of thickness $e$ made of such effective medium and sandwiched between the substrate and the air, the component of the wavenumber along $x_{2}$ in the layer is

$$
\delta=\sqrt{\varepsilon_{\|}\left(k^{2}-\beta^{2} / \varepsilon_{\perp}\right)},
$$

being the equivalent of the component $\gamma$ in the substrate, (25), and the reflection coefficient $\tilde{r}$ reads as

$$
\left\{\begin{aligned}
\tilde{r} & =\frac{\left.i \tan (\delta e)\left(1-\xi_{1} \xi_{2}\right)+\left(\xi_{1}-\xi_{2}\right)\right)}{i \tan (\delta e)\left(1+\xi_{1} \xi_{2}\right)-\left(\xi_{1}+\xi_{2}\right)}, \\
\text { with } \quad \xi_{1} & =\varepsilon_{\|} \frac{\alpha}{\delta}, \quad \xi_{2}=\frac{\varepsilon_{\|}}{\varepsilon} \frac{\gamma}{\delta} .
\end{aligned}\right.
$$

While this classical homogenization leads to accurate results when $e$ is large, which means of the order of magnitude or larger than the wavelength, it is not efficient to predict the scattering properties of a layer with $k e \ll 1$. We shall illustrate this failure in the following section.

\section{NUMERICAL AND EXPERIMENTAL VALIDATION}

\section{A. Realization and characterization of the sample and ellipsometric measurements}

A grating was realized on a Si substrate using optical lithography. The pattern was obtained using Direct Writing Lithography with a focused laser beam at $405 \mathrm{~nm}$ defining the lines in a positive resist. This process allowed achieving a linewidth resolution of approximately $1 \mu \mathrm{m}$ over an area of $1 \times 2 \mathrm{~cm}^{2}$. After development, the Si was etched using Reactive Ion Etching (RIE), with a limited process time so that vertical walls were obtained.

The measurements of the scattering properties were performed on a Woollam WVASE ellipsometer. Ellipsometry measures the ratio of the reflexion coefficients $r_{\mathrm{TM}}$ in TM polarization and $r_{\mathrm{TE}}$ in TE polarization, specifically

$$
r=\frac{r_{\mathrm{TM}}}{r_{\mathrm{TE}}}=\tan \psi e^{i \varphi},
$$

with $\varphi=\varphi_{\mathrm{TM}}-\varphi_{\mathrm{TE}}$ the phase shifts after reflexion in TM and TE polarizations, and $\Psi=$ atan $\left(\left|r_{\mathrm{TM}} / r_{\mathrm{TE}}\right|\right)$ the so-called ellipsometric angle. The ellipsometer was equipped with a Fourier Transform InfraRed spectrometer as a source. The measurements were performed in the 2-20 $\mu \mathrm{m}$ range with a resolution of $32 \mathrm{~cm}^{-1}$ and at different angles selected as to follow the ellipsometric angles evolution across the Brewster's angle. In the following, we shall analyze the results for $\lambda>5 \mu \mathrm{m}$, below which higher interference orders appear, leading to Rayleigh Wood anomalies. In fact, the interference order -1 appears in the substrate at $\lambda=9 \mu \mathrm{m}$, but we checked numerically that this does not affect significantly the 0 order.

Eventually, after the measurement were done, the sample was cleaved to allow for accurate measurements of the grating profile in Scanning Electron Microscopy (SEM), see Fig. 1. From the different realized SEM profiles, the shape of the periodic roughnesses appears to be almost rectangular, of width $0.86 \mu \mathrm{m}$ for a grating periodicity $h=2.0 \mu \mathrm{m}$, whence $f=0.43$, and of depth $0.313 \mu \mathrm{m}$. For this geometry, and with $\varepsilon_{\mathrm{r}}=11.7$, we computed $(\mathcal{B}, \mathcal{C})$ defined by (23) and entering in the jump conditions (22) by solving the elementary problems (16) and (17), and found

$$
\mathcal{B}=0.519, \quad \mathcal{C}=0.061,
$$

which provide the reflection coefficient in (26) without any ajustable parameter. For the layer model, we have from (29) that $\varepsilon_{\|}=0.607$ and $\varepsilon_{\perp}=0.178$, from which the reflection coefficient in (31) is known (also without any ajustable parameter).

\section{B. Typical measurements of the reflection, the pseudo Brewster angle}

From now on, we shall use the angles $(\psi, \varphi)$ to characterize the modulus and the phase of the reflection coefficient. For reference calculations, we use direct numerics in TM and TE polarizations using a modal method similar to the RCWA [12]. Finally, to avoid additional discussion of the models, we use $(r, \tilde{r})$ in Eqs. (26) and (31) from the models in TM polarization, and the reflection coefficient given by the modal calculation in TE polarization.

We start by reporting in Fig. 3 typical variations of $\psi$ and $\varphi$ as a function of $\theta$ (for $\lambda=5,10,15$ and $20 \mu \mathrm{m}$ ). The experimental measurements (open circles) are compared to the modal calculations (dashed lines) and to (i) the interface model (26) (plain lines) and (ii) the layer model (31) (dotted lines). As expected, the agreement between the modal calculations and the experiments is excellent. Next, the accuracy of the interface model is very good without any ajustable parameter, and expectedly it is better and better for larger and larger wavelengths. Note that this is a good news since our frequency range did not fulfilled $a$ priori the low frequency regime corresponding to $\eta=k h \ll 1$ : indeed, with $h=2 \mu \mathrm{m}$, wavelengths $\lambda=5,10,15,20 \mu \mathrm{m}$ correspond to $\eta=2.5,1.3,0.8,0.6$, which 
reveals an unexpected robustness of the interface model. In comparison, the layer model, without adjustable parameter, predicts significantly larger shifts of the Brewster effect than the measured ones. This overestimate of the effect of the roughnesses is confirmed using classical retrieval procedures based on EMA models (Effective Medium Approximation) of the Bruggeman type. In this case, the set of measured reflection coefficients is used in an inverse problem to retrieve $(e, f)$. Assuming in the EMA an equivalent isotropic layer filled with small spheres, the retrieved thickness is $e=0.256 \mu \mathrm{m}$, and assuming an equivalent anisotropic layer filled with stems, the retrieved thickness is $e=0.239 \mu \mathrm{m}$, thus about $20 \%$ smaller than the actual thickness (for both EMA, the filling fraction is correctly found, with $f \simeq 0.44$ ).

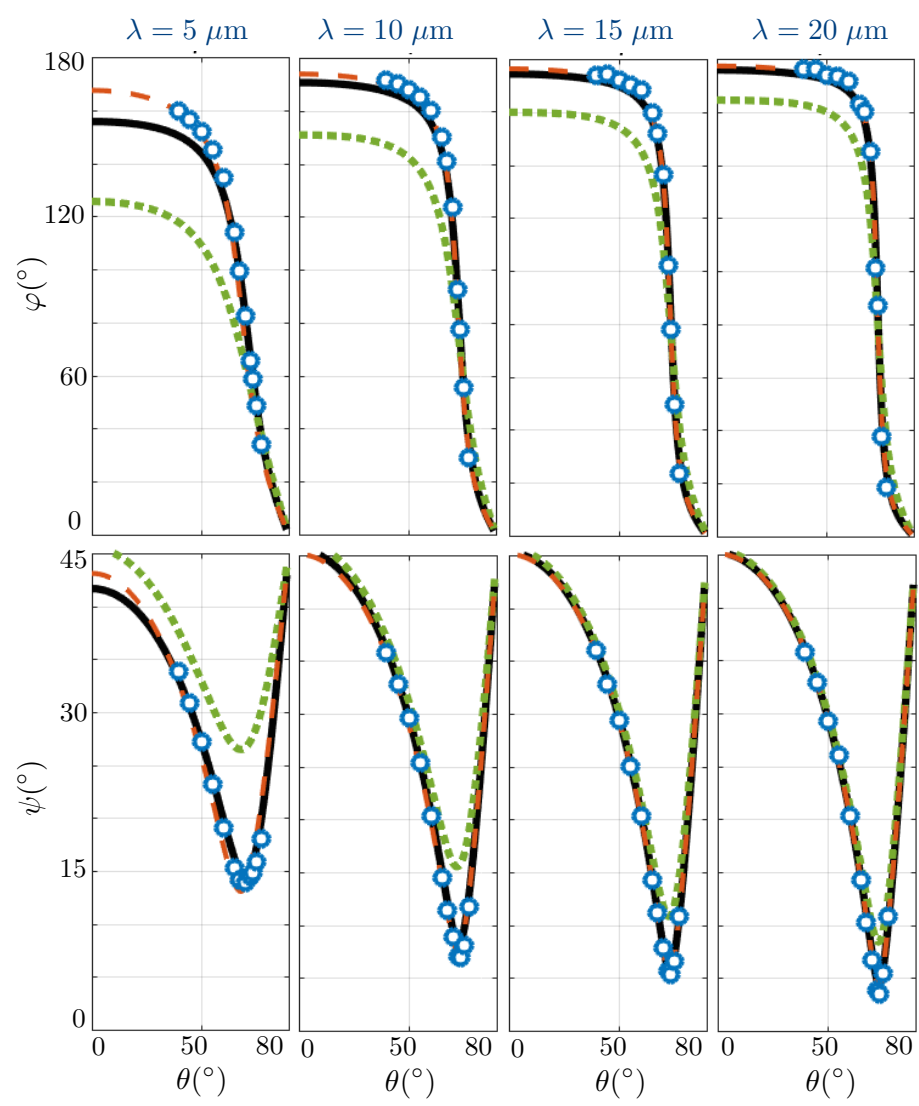

Fig. 3. Variation of $\psi$ and $\varphi$ as a function of the incidence $\theta$ for $\lambda=5,10,15$ and $20 \mu \mathrm{m}$. Experimental measurements (blue open symbols), direct modal calculation (red dotted lines), interface model (26) (black plain lines) and classical layer model (31) (green dotted lines). For the interface and layer models, we used the actual dimensions of the sample $e=0.313 \mu \mathrm{m}, h=2 \mu \mathrm{m}$ and $f=0.43$ (without ajustable parameters).

\section{Characterization of the shift in the Brewster effect}

From the profiles of $(\psi, \varphi)$, it is possible to quantify the shift in the Brewster effect. We report in Fig. 4 the minimum in the reflection modulus as a function of $\lambda$ (to find such minimum from the experimental profiles in Figs. 3, we used a spline interpolation of the data). Again, the modal calculations and the experimental results are in good agreement. The prediction from our interface model for $\lambda>5$ $\mu \mathrm{m}$ is rather good when compared to both the numerics and the experiments, and as previously said, it is more accurate for larger wavelength; specifically, the discrepancy with the experimental results ranges between 0.3 and $1.4^{\circ}$, corresponding to relative errors of 5 to $20 \%$. Finally, the minimum of reflection follows reasonably a power law in $1 / \lambda$ according to our estimate of $r_{\text {min }}$ ( (28) (this latter is reported in dashed-dotted line, and the relative better agreement with the experiments is casual). In comparison, the layer model predicts a much higher reflection, with $\psi$ being about twice the actual one (and with a discrepancy ranging from 5 to $10^{\circ}$, the relative error is always larger than $100 \%$ ).

We now move on a more subtil effect, which is the deviation in the Brewster angle $\theta_{\mathrm{B}}$ to the pseudo Brewster angle $\theta_{\mathrm{PB}}$. In Figs. 5 , we considered two different estimations of $\theta_{\mathrm{PB}}$, namely (i) the angle at the minimum of the reflection modulus min $(\psi)$ and (ii) the angle realizing $\varphi=90^{\circ}$ (and to avoid multiple notations, we term both $\theta_{\mathrm{PB}}$ ). Obviously, the first criterion is intuitively the more adapted to measure the shift with respect to a vanishing reflection. On the other hand, the second criterion is often used to characterize a sudden change in the scattering response, as resonance phenomena, and it has the advantage to be very precise since the large variation in the reflection phase occurs in a very short angle range (this is visible in Figs. 3). This is indeed what we observe, with a dispersion in the results coming from the experimental data much more important in Fig. 5(a) than in Fig. 5(b). Although the interface model captures the variations in $\theta_{\mathrm{B}}-\theta_{\mathrm{PB}}$ more accurately than the layer model, this agreement remains qualitative. Indeed, the deviation in the Brewster angle follows (in both cases) a $1 / \lambda^{2}$ law for $\lambda>8 \mu \mathrm{m}$. This is predicted from our interface model since (26) does not 


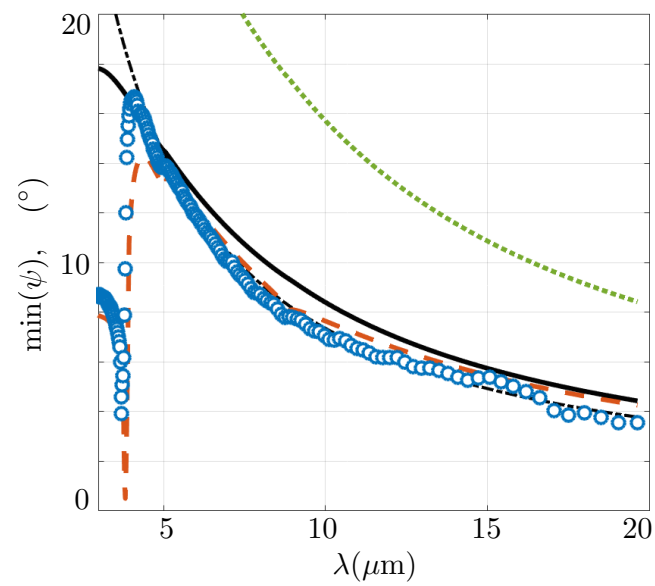

Fig. 4. Minimum of the reflection modulus $\psi$ as a function of $\lambda$. From the experimental measurements (open symbols), the modal calculations (red dotted lines), the interface model (26) (black plain lines, the black dashed-dotted shows the approximated result, (28)) and the classical layer model (31) (green dotted lines).

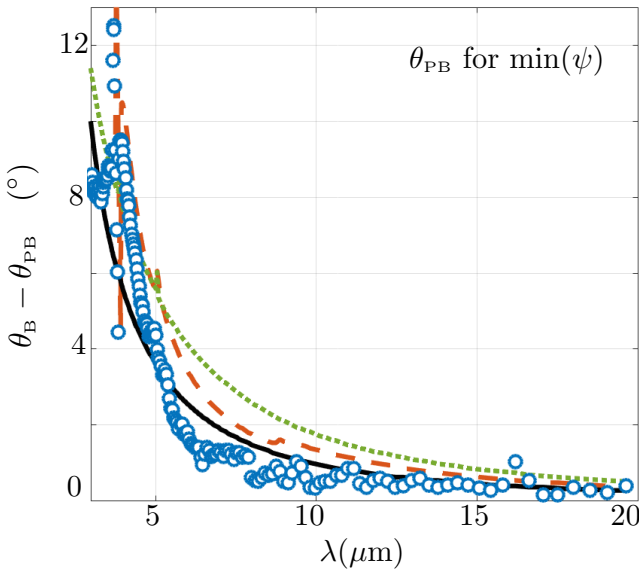

(a)

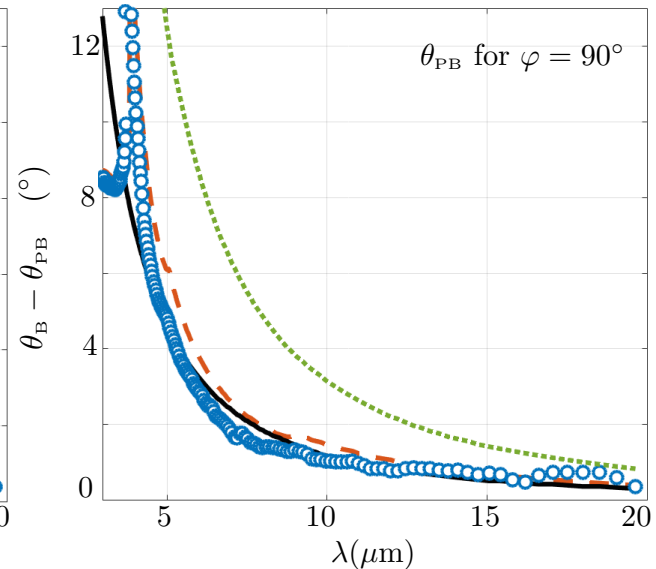

(b)

Fig. 5. Deviation $\theta_{\mathrm{B}}-\theta_{\mathrm{PB}}$ of the Brewster angle as a function of the wavelength, (a) at the minimum in $\psi$ and (b) at $\varphi=90^{\circ}$. Same representation as in Fig. 4.

predict a shift at the order $O(\eta)=O(1 / \lambda)$, but we reach the limit of validity of the model derived up to $O\left(\eta^{2}\right)$ (to get quantitative predictions, one should derive the model up to $\left.O\left(\eta^{3}\right)\right)$. Incidentally, although the two deviations look similar, they differ in $O\left(1 / \lambda^{2}\right)$ law, which means that the angles realizing the minimum of reflection and realizing the $90^{\circ}$ - reflection phase differ at the dominant order.

\section{NUMERICAL STUDY OF THE INFLUENCE OF THE ROUGHNESS THICKNESS}

We have stated and illustrated that the interface model is adapted to small roughness thicknesses $(k e \ll 1)$ while the layer models, as the Maxwell-Garnett model, the EMA or the model of layered medium presented in the Section 3.B are adapted in the opposite limits $(k e \gg 1)$. In this section, we show in more details what is happening when increasing the roughness thickness. To do so, we use comparison of the models with direct numerics; for simplicity, we consider $r_{\mathrm{TM}}$ which is available from the direct numerics, and which is the prediction given in the models, Eqs. (26) and (31).

We computed the variations of $r_{\mathrm{TM}}$ as a function of the incidence $\theta$ for a fixed frequency corresponding to $k h=0.5$ and increasing the value of $e / h$. Results are reported in the top panels of Figs. 6 . We observe 3 behaviors: for small thicknesses $e / h$, the interface model is the most accurate $(e / h=0.1)$ and we shall be more explicit since at this stage, this is not obvious by simple inspection of the plot. For intermediate $e / h$ values, both models experience a transition : the layer model becomes more and more accurate while the interface model becomes less and less accurate $(e / h=0.6$ and 1.6). Eventually, for large $e / h$ values, the layer model becomes clearly the most accurate $(e / h=4)$.

From the top panels of the Figs. 6, the layer model appears reasonably predictable for all $e / h$ values if we are interested in the overall behavior of the reflection over the range $\left(0,90^{\circ}\right)$. The reason is simple: because the effect of the roughnesses vanishes for vanishing $e / h$, the reflection for small $e / h$ is merely the same as for a flat interface, and this is obtained in both models. But if we are precisely interested in the small variations with respect to the flat case, we want to be predictable when they are the most significant, 


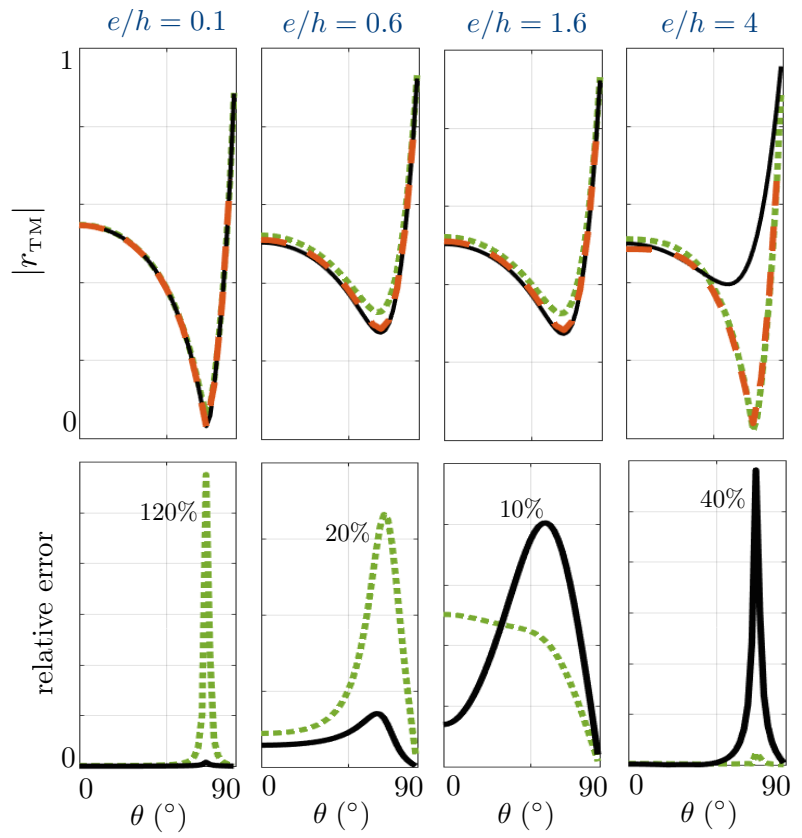

Fig. 6. Comparison of the layer and interface models for increasing $e / h$ values. The top panels report the variations of $\psi$ as a function of $\theta$ (open symbols: direct numerics, plain lines: interface model, dashed lines: layer model. The bottom panels reports the relative errors in $\psi$ in the two models.

that is near the Brewster angle. The bottom panels in Fig. 6 show the relative errors, which allows to specify the accuracy of the models. For $e / h=0.1$, the layer model appears to be unable to describe quantitatively the small shift in $r_{\mathrm{TM}}$ near the Brewster angle, reaching an error larger than $100 \%$; outside this region, the error does not exceed $2 \%$ but this small discrepancy is precisely of the same order of magnitude than the small shift with respect to the flat case. To the contrary, the interface model keeps a very small error over the whole range $\left(0,90^{\circ}\right)$, with a maximum of $1.5 \%$ at the Brewster angle, otherwise less than $0.1 \%$. Next increasing $e / h$ produces an increasing effect of the propagation effect against the boundary layer effect, and the error in the layer model becomes more and more accurate while the interface model becomes less and less accurate.

\section{CONCLUSION}

In this work, we have proposed and validated an interface model able to describe accurately the scattering of light by periodically roughnesses with small thickness. The typical range of validity of the model is $k h<1$ and $e / h<1$, although we observed that the model remains surprisingly predictive up to the first Rayleigh-Wood anomaly (in the experiments at $\lambda=5 \mu \mathrm{m}$ ). In comparison, classical layer models which consist in replacing the roughnesses by a layer filled with an effective medium are not predictive at all when the direct problem is sought.

Of course, retrieval methods can be used in which an effective thickness and an effective filling fraction are used as free parameters. However, the use of such methods is questionable if the retrieved parameters are far from the actual ones and we have seen that the retrieved thickness was significantly underestimated in our experiments (about 20\%). This is why we think that the use of such layer models is unadapted. Indeed, once a retrieved thickness has been obtained for a given sample, it is not obvious that doubling the sample thickness leads to doubling the effective thickness, and if it is not the case, the retrieval method becomes simply ad hoc since it does not allow to anticipate other sample responses. More generally, tempting a model in a retrieval procedure without having validated the model in the direct problem is hazardous; it may lead to multiple local minima in the space of the retrieved parameters which simply reflect the inadequation between the real system and the model that we impose to it.

\section{ACKNOWLEDGMENTS}

The authors thanks Kim Pham for fruitful discussions. A.M. and J.-J.M. acknowledge the financial support of the french Mission Interdisciplainare du Centre National de la Recherche Scientifique (MI/CNRS) under grant INFYNITI/PomS.

\section{REFERENCES}

1. M. Saillard and D. Maystre, "Scattering from metallic and dielectric rough surfaces," JOSA A 7, 982-990 (1990).

2. M. Saillard, "A characterization tool for dielectric random rough surfaces: Brewster's phenomenon," Waves in random media 2, 67-79 (1992).

3. J.-J. Greffet, "Theoretical model of the shift of the brewster angle on a rough surface," Optics letters 17, 238-240 (1992).

4. C. Baylard, A. A. Maradudin, and J.-J. Greffet, "Coherent reflection factor of a random rough surface: applications," JOSA A 10, $2637-2647$ (1993).

5. A. Maradudin, R. Luna, and E. Méndez, "The brewster effect for a one-dimensional random surface," Waves in Random Media 3, 51-60 (1993). 


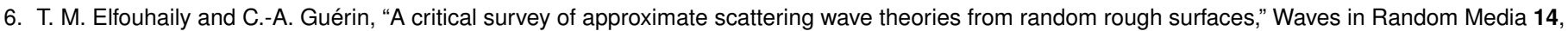
R1-R40 (2004).

7. J. Nevard and J. B. Keller, "Homogenization of rough boundaries and interfaces," SIAM Journal on Applied Mathematics 57, 1660-1686 (1997).

8. G. Kristensson, "Homogenization of corrugated interfaces in electromagnetics," Progress in electromagnetics research 55, 1-31 (2005).

9. A. H. Sihvola, Electromagnetic mixing formulas and applications, 47 (let, 1999).

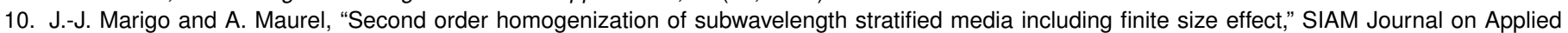
Mathematics 77, 721-743 (2017).

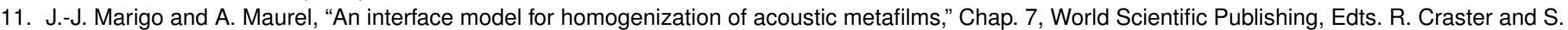
Guenneau (2017).

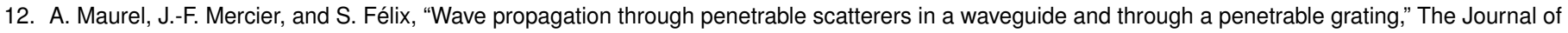
the Acoustical Society of America 135, 165-174 (2014).

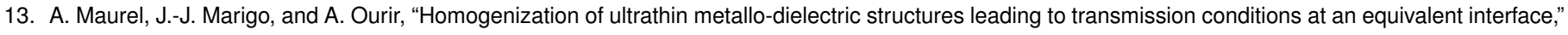
The Journal of the Optical Society of America B 33, 947-956 (2016).

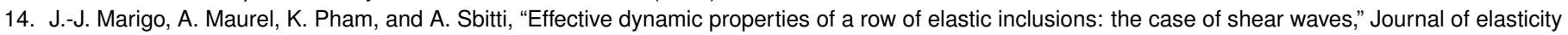
(2017).

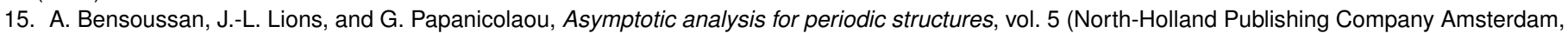
1978).

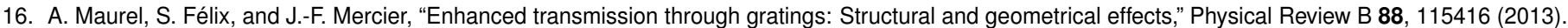

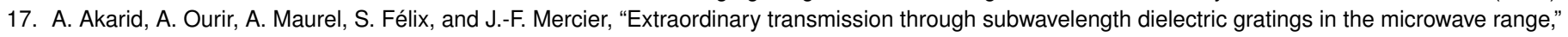
Optics letters 39, 3752-3755 (2014). 